\title{
Reemplazo parcial de rodilla mediante el sistema robótico NAVIO: resultados clínicos postquirúrgicos evaluados mediante Knee injury Osteoartritis Outcome Score
}

\section{Robotic-Assisted Unicompartmental Knee Replacement with NAVIO Surgical System: Outcome Evaluation using the Knee Injury Osteoarthritis Outcome Score}

Pablo D. Vega Parra ${ }^{1} \quad$ J. Dionisio Palacios Barajas ${ }^{2} \quad$ Raúl A. Márquez Ambrosi ${ }^{3} \quad$ Jorge R. Duarte ${ }^{4}$

${ }^{1}$ Estudiante de Medicina, Universidad Adventista de la Plata, Entre Rios, Argentina

2 Fellowship de Ortopedia y Traumatología Deportiva, Reconstrucción Articular y Cirugía Ortopédica Robótica, Marquez Robotic Orthopedic Institute, Edinburg, Texas, United States

${ }^{3}$ Director en Reconstrucción Articular, Ortopedia y Traumatología Deportiva y Cirugía Ortopédica Robótica, Marquez Robotic Orthopedic Institute, Texas, United States

${ }^{4}$ Médico, Marquez Robotic Orthopedic Institute, Edinburg, Texas, United States

Address for correspondence Pablo Vega, MD, Victor Rae 4957, Las Condes, RM, Codigo Postal 7580565, Santiago, Chile; 3808 Oak Ridge, zip code 77523, Baytown, TX, United States (e-mail: pablovegap@gmail.com).

Rev Chil Ortop Traumatol 2017;58:7-12.

\section{Resumen \\ Palabras clave \\ - Reemplazo Parcial De Rodilla \\ - procedimiento quirúrgico robótico \\ - resultados evaluados por los pacientes}

Introducción La osteoartritis de rodilla es una de las patologías musculo esqueléticas más prevalentes en el mundo, y el tratamiento quirúrgico es hasta hoy, la única terapia definitiva. El sistema $\mathrm{NAVIO}^{\mathrm{TM}}$ es uno de los dispositivos robóticos más recientemente a disposición para realizar el reemplazo parcial de rodilla. Ofrece gran precisión en el proceso de alineamiento, remodelado óseo y balance de los tejidos blandos, sin exponer al paciente a la radiación ni a los costos de la tomografía computarizada que suele realizarse con los sistemas robóticos actuales antes del procedimiento artroplástico.

Objetivo Evaluar los resultados medidos por los pacientes antes y después del procedimiento artroplástico mediante $\mathrm{NAVIO}^{\mathrm{TM}}$ y la prótesis STRIDETM .

Material y Método Aplicación del cuestionario Knee injury Osteoarthritis Outcome Score (KOOS) en el preoperatorio y a los 12 meses del procedimiento quirúrgico de los primeros 47 pacientes operados usando $\mathrm{NAVIO}^{\mathrm{TM}}$ en Cornerstone Regional Hospital, Edinburg, Texas. received

November 15, 2016 accepted

December 2, 2016
DOI http://dx.doi.org/ 10.1055/s-0037-1599200. ISSN 0716-4548.
Copyright (e 2017 by Thieme-Revinter

Publicações Ltda, Rio de Janeiro, Brazil
License terms

c) $(1) \$$ 


\section{Abstract}

\section{Keywords}

- unicompartimental knee arthroplasty

- robotic surgical procedure

- outcome measures
Resultados Se observó una mejoría de las muestras normalizadas en todas las categorías con un $p<0.05$.

Comentario Estudios recientes han sugerido que en Chile la presentación de la osteoartritis se asocia a un grado de severidad menor que en el resto de los países latinoamericanos. Lo cual torna relevante la aplicación de terapias quirúrgicas más conservadoras, que ofrezcan al paciente un alivio sintomático y le permitan reestablecerse a sus actividades cotidianas causando el mínimo impacto posible. Esas ventajas fueron observadas en los pacientes sometidos a ese procedimiento quirúrgico.

Conclusiones La artroplastia unicompartimental de rodilla mediante el sistema quirúrgico $\mathrm{NAVIO}^{\mathrm{TM}}$ y prótesis STRIDE ${ }^{\mathrm{TM}}$ demostró mejorar substancialmente la vida diaria y calidad de vida de los pacientes, disminuyendo el dolor y mejorando su función durante las actividades deportivas y recreacionales.

Introduction Knee osteoarthritis is one of the most prevalent musculoskeletal pathologies in the world and the surgical treatment is until today the only definitive therapy. $\mathrm{NAVIO}^{\mathrm{TM}}$ system is one of the most recently available robotic devices for partial knee arthroplasty. It offers great precision in the process of alignment, bone remodeling and soft tissue balancing without exposing the patient to the radiation or the costs of the $\mathrm{CT}$ scan usually performed before the arthroplasty with the current robotic systems.

Objective To evaluate the outcomes measured by the patients before and after the arthroplasty using $\mathrm{NAVIO}^{\mathrm{TM}}$ and the STRIDE ${ }^{\mathrm{TM}}$ prosthesis.

Materials and Methods Application of the Knee Injury Osteoarthritis Outcome Score (KOOS) questionnaire in the preoperative and 12 months after the surgical procedure of the first 47 patients operated using $\mathrm{NAVIO}^{\mathrm{TM}}$ at Cornerstone Regional Hospital, Edinburg, Texas.

Results There was an improvement of the normalized samples in all the categories with $p<0.05$.

Commentary Recent studies have suggested that in Chile the presentation of osteoarthritis is associated with a lower degree of severity compared with the rest of Latin American countries. This makes relevant the application of more conservative surgical therapies, which could offer the patient symptomatic relief and allow them to reestablish their daily activities causing the minimum impact possible. These advantages were observed in patients undergoing this surgical procedure.

Conclusions Unicompartmental knee replacement using NAVIO TM surgical system and STRIDE ${ }^{\mathrm{TM}}$ prosthesis demonstrated a substantial improvement in patient's quality of life, reducing pain and improving their function during sports and recreational activities.

\section{Introducción}

La osteoartritis de rodilla es una de las patologías músculo esqueléticas más prevalentes en el mundo ${ }^{1} \mathrm{y}$ es un factor preponderante en la disminución de la calidad de vida poblacional. $^{2}$ Genera un impacto económico negativo substancial, debido en parte al incremento de los Años Vividos con Discapacidad (AVD) que conlleva, ${ }^{3}$ y por los crecientes costos que derivan de su tratamiento a corto y largo plazo. ${ }^{4}$ De hecho, se estima que para el año 2030 en los Estados Unidos, los reemplazos de rodilla aumentarán en demanda un $673 \%$ en relación al año $2005 .^{5}$
El reemplazo parcial de rodilla (RPR) ha demostrado muy buenos resultados a través de décadas ${ }^{6}$; pero las recomendaciones y percepciones a respecto de su uso continúan controversiales. $^{7-9}$

Los Resultados Medidos por los Pacientes (del inglés Patient Related Outcome Measures o PROM) se pueden definir como: "Cualquier información sobre el estado del paciente relacionado a su condición médica, la evolución de su salud, su percepción sobre la asistencia médica recibida o tras la aplicación de una medida de salud; obtenida directamente del paciente, sin modificaciones por el personal médico u otro profesional de salud." ${ }^{10}$ Los PROMs son colectados 
generalmente mediante encuestas auto administradas, cuyo objetivo es evaluar la percepción del paciente en relación a su salud. Su aplicación ha demostrado que no siempre el procedimiento más tradicional (en este caso, el reemplazo total de rodilla) está relacionado con una mejoría en la calidad de vida del paciente, aún cuando se ofrece un resultado "ortopédicamente correcto." ${ }^{11}$ Esas discrepancias encontradas dejan en evidencia la necesidad de continuar investigando en esa área para poder llegar a conclusiones relevantes $^{12,13}$; especialmente debido al incremento en el rol que tienen los PROM en la toma de decisiones de organizaciones nacionales de salud y como herramienta para evaluar la eficacia de procedimientos e intervenciones. ${ }^{14}$

En pacientes que cumplen con los criterios para un RPR, elegir esta alternativa puede ofrecer beneficios en comparación al reemplazo total de rodilla (RTR), siendo las más evidentes: la conservación de una mayor porción de la anatomía normal de la rodilla, y el menor daño a los tejidos del paciente. Además, se ha observado que existe un mayor porcentaje de pacientes que pueden retornar a sus actividades deportivas tras un RPR en comparación a los sometidos a un RTR. ${ }^{15,16}$ El RPR ha presentado una proporción significativamente mayor de pacientes que después del procedimiento consiguen flexionar sus rodillas a $120^{\circ} \mathrm{o}$ más, ${ }^{17}$ y existe evidencia que sugiere una mejoría en la marcha después de un RPR casi comparable a la marcha normal, siendo levemente superior a los resultados pos RTR. $^{18}$

\section{Material y Método}

Se incluyeron a los 47 pacientes sometidos a un RPR de compartimento medial mediante el sistema robótico NAVIO $^{\mathrm{TM}}$ de Smith \& Nephew, durante el periodo compuesto entre el 5 de noviembre del 2013 y el 30 de febrero del 2014, en Cornerstone Regional Hospital, Edinburg, Texas. Los criterios de inclusión usados fueron: Ligamento cruzado anterior intacto, ausencia de deformidad $>10^{\circ}$ de contractura en flexión, artrosis patelofemoral de leve a moderada sin síntomas relacionados al compartimento anterior ni al compartimento lateral. Se realizaron las siguientes radiografías: anteroposterior en carga, posteroanterior en flexión en carga, lateral de rodilla y axial de rótula. No se solicitaron radiografías en estrés, ya que la corrección definitiva se realiza por medio de la liberación de los tejidos blandos y retiro de osteofitos del compartimento medial. Se decidió la prótesis unicompartimental por la sintomatología del paciente y la disminución del espacio articular del compartimento medial observado en la radiografía posteroanterior con la rodilla en flexión.

Todos los pacientes fueron operados por el mismo cirujano ortopédico, y se usó la prótesis STRIDETM de Smith \& Nephew.

Aunque la conversión intraoperatoria a un RTR es siempre una posibilidad, no fue necesaria en ninguno de los casos estudiados. Tampoco hubo necesidad de intervenir mediante una revisión quirúrgica en ninguno de los casos durante el periodo de tiempo del estudio.

\section{Sistemas robóticos y de navegación aplicados a procedimientos artroplásticos}

Los sistemas de navegación han aumentado la precisión en el proceso de alineamiento del miembro afectado, ${ }^{19-21}$ y los sistemas de asistencia robótica ofrecen ventajas al momento de remodelar el hueso y balancear los tejidos blandos. ${ }^{22,23}$ Sabiendo que alineación e impacto en los tejidos son dos variables fundamentales para el éxito del procedimiento, ${ }^{24,25}$ es posible apreciar el beneficio de usar sistemas modernos de navegación computarizada en comparación a los métodos convencionales. ${ }^{26}$

\section{Solución Robótica NAVIO'm (de "Smith \& Nephew")}

NAVIO $^{\mathrm{TM}}$ de Smith \& Nephew (tras la adquisición de Blue Belt Technologies a fines del año 2015), además de integrar las características positivas relacionadas a la elección del RPR y navegación computarizada anteriormente descritas; y gracias a su tecnología patentada de mapeo articular ${ }^{27}$ ofrece la ventaja de no exponer al paciente a la radiación y a los costos de la tomografía computarizada, sin afectar la precisión del sistema ${ }^{23,28}$; marcando así una diferencia técnica en comparación a otros sistemas robóticos disponibles actualmente, debido a que suelen requerir una tomografía previa al procedimiento quirúrgico.

Este sistema obtiene un mapeo de la superficie articular a ser modelada a través de seguimiento infrarrojo, y calcula en tiempo real la alineación articular. Toda esa información es presentada al cirujano de manera instantánea mediante la generación virtual de un modelo 3D, permitiéndole tomar decisiones dinámicas intraoperatorias. Luego, con el dispositivo robótico de mano, el cirujano puede remodelar con gran precisión la superficie donde será implantada la prótesis, gracias a la retracción o protrusión robótica de la broca que está contenida en el dispositivo manual. Eso le permite obtener una exactitud milimétrica en la remodelación ósea, y una conservación máxima de la anatomía normal del paciente. ${ }^{29}$

El sistema NAVIO ${ }^{\mathrm{TM}}$ ofrece también la facilidad técnica de ser una plataforma abierta dónde pueden ser utilizadas prótesis de terceros. Así, el cirujano puede trabajar con la prótesis que desee, no estando limitado a utilizar el sistema protésico recomendado por el fabricante.

La rápida adaptación del cirujano a ese sistema ha sido comprobada, permitiéndole tras tan solo 5 procedimientos, realizar toda la cirugía en una media de 48 minutos. Sin comprometer la precisión en la realización del proceso quirúrgico. $^{30}$

A la fecha de este estudio, es uno de los dispositivos más recientemente incluidos en el campo de las artroplastias unicompartimentales de rodilla; habiendo recibido autorización por la FDA para su uso en los Estados Unidos de América a mediados del año 2012. ${ }^{31}$

El sistema protésico STRIDE UNI knee system de Smith \& Nephew, ha sido desarrollado y optimizado para trabajar junto con el sistema robótico $\mathrm{NAVIO}^{\mathrm{TM}}$. Es una solución protésica creada para los RPR, enfocada en conservar al 
máximo el tejido óseo del paciente. Los beneficios descritos por el fabricante son: componente femoral diseñado para adaptarse a una flexión acentuada; la porción posterior del componente femoral es curvo, lo que permite una menor resección de hueso; platillo tibial redondeado, y ofrecido en tamaños anatómicamente correspondientes. ${ }^{32}$

Es importante resaltar que in vitro se ha demostrado gran variabilidad en el desgaste prostético al variar su diseño y composición, ${ }^{33,34}$ por lo cual la elección de la prótesis puede ser un factor relevante al evaluar los resultados del procedimiento artroplástico.

\section{Cuestionario Knee Injury Osteoarthritis Outcome Score (KOOS)}

En este estudio se usó el índice KOOS aplicando el cuestionario correspondiente ${ }^{35}$ en la evaluación preoperatoria y durante el control a los 12 meses del procedimiento quirúrgico.

El KOOS fue creado simultáneamente en inglés y alemán suizo, pero ha sido validado en varios idiomas inclusive en español ${ }^{36}$; el cual se aplicó para evaluar la evolución de los sujetos elegidos en este estudio. Las instrucciones de puntuación, el cuestionario y la hoja de cálculo para realizar la puntuación fueron provistas gratuitamente en la página web de los creadores del índice KOOS. ${ }^{37}$

El cuestionario, auto administrado, está compuesto por 42 preguntas divididas en 5 categorías: "síntomas," "dolor," "actividades cotidianas," "función, actividades deportivas y recreacionales" y "calidad de vida." Cada pregunta tiene 5 opciones de respuesta según la cual le es asignado un valor del 0 al 4 . Un puntaje normalizado es definido para cada categoría, siendo 100 definido como ningún síntoma y 0 síntomas extremos. Con los puntajes de cada una de las categorías puede ser generado un perfil del resultado de cada paciente.

\section{Resultados}

Del total de la muestra $(n=47), 23$ sujetos eran de sexo femenino (49\%) y 24 de sexo masculino (51\%). La media de edad de los pacientes al momento de la cirugía fue de 67 años (45-77 años). El $100 \%$ del total de la muestra $(n=47)$ fue sometido al procedimiento quirúrgico artroplástico mono articular de compartimento medial por el mismo cirujano, mediante $\mathrm{NAVIO}^{\mathrm{TM}}$ y usando la prótesis STRIDE.

Con un nivel de confiabilidad del 95\% podemos afirmar que todas las categorías evaluadas según el intervalo que se muestra en la Tabla 1, contienen el valor de la media muestral, con un valor $p<0,05$.

Podemos decir entonces que todas las categorías evaluadas mejoran significativamente posteriormente al procedimiento artroplástico mediante el sistema robótico $\mathrm{NAVIO}^{\mathrm{TM}} \mathrm{y}$ usando la prótesis STRIDE.

\section{Frecuencia y distribución en las 5 categorías evaluadas mediante el KOOS pre y pos quirúrgico}

\section{Categoría: Síntomas}

La categoría Síntomas en la evaluación pre quirúrgica posee una media considerablemente baja $(\mathrm{M}=33,11$; $\mathrm{DE}$ $=1,531$ ), siendo 100 definido como ningún síntoma y 0 síntomas extremos, la mayor cantidad de puntajes se aglomeran en los valores superiores a la media, mientras que en la evaluación pos quirúrgica esa categoría posee una media casi dos veces más alta $(\mathrm{M}=70,79 ; \mathrm{DE}=1,060)$.

\section{Categoría: Dolor}

La categoría Dolor en la evaluación pre quirúrgica tiene una media considerablemente baja, $(\mathrm{M}=35,30 ; \mathrm{DE}=6,78)$. La mayor cantidad de puntajes se aglomeran en los valores menores a la media. En la evaluación pos quirúrgica esta categoría posee una media más alta $(\mathrm{M}=71,62 ; \mathrm{DE}=, 814)$.

\section{Categoría: Actividades cotidianas}

La categoría Actividades cotidianas en la evaluación pre quirúrgica tiene una media baja, $(\mathrm{M}=35,23 ; \mathrm{DE}=, 712)$. La mayor cantidad de puntajes se aglomeran en los valores mayores a la media. En la evaluación pos quirúrgica esta categoría posee una media alta $(\mathrm{M}=71,47 ; \mathrm{DE}=, 821)$.

\section{Categoría: Función, actividades deportivas y recreacionales}

La categoría Función, actividades deportivas y recreacionales en la evaluación pre quirúrgica tiene una media

Tabla 1 Resultados de la aplicación del cuestionario KOOS: Comparación pre y pos quirúrgica de la media, intervalo de confianza de la media, desvío estándar y valor $p$

\begin{tabular}{|l|l|l|l|l|l|}
\hline \multirow{2}{*}{} & \multicolumn{2}{|l|}{ Preoperatorio } & \multicolumn{2}{l|}{ Postoperatorio } \\
\cline { 2 - 6 } & M (IC) & DE & M (IC) & DE & \\
\hline Medias & $32,68(31,28-34,08)$ & 0,697 & $70,06(68,72-71,41)$ & 0,669 & $<0,05$ \\
\hline Síntomas & $33,11(30,03-36,19)$ & 1,531 & $70,79(68,65-72,92)$ & 1,060 & $<0,05$ \\
\hline Dolor & $35,30(33,31-37,29)$ & 6,779 & $71,62(69,98-73,26)$ & 0,814 & $<0,05$ \\
\hline Actividades cotidianas & $35,23(33,80-36,67)$ & 0,712 & $71,47(69,82-73,12)$ & 0,821 & $<0,05$ \\
\hline Actividades deportivas y recreacionales & $28,51(26,68-30,35)$ & 0,911 & $63,62(60,92-66,32)$ & 1,341 & $<0,05$ \\
\hline Calidad de vida & $31,15(28,65-33,65)$ & 1,241 & $72,98(71,00-74,96)$ & 0,983 & $<0,05$ \\
\hline
\end{tabular}

Abreviaciones: DE, desviación estándar; KOOS, Knee injury Osteoarthritis Outcome Score; IC, intervalo de confianza; M, media. 
notablemente baja, ( $\mathrm{M}=28,51$; $\mathrm{DE}=$, 911). La mayor cantidad de puntajes están aglomerados en los valores mayores a la media. En la evaluación pos quirúrgica esa categoría posee una media alta $(\mathrm{M}=63,62 ; \mathrm{DE}=1,341)$.

\section{Categoría: Calidad de Vida}

La categoría Calidad de vida en la evaluación pre quirúrgica tiene una media baja, $(\mathrm{M}=31,15 ; \mathrm{DE}=1,241)$. La mayor cantidad de puntajes están aglomerados en los valores menores a la media. En la evaluación pos quirúrgica esta categoría posee una media alta $(\mathrm{M}=72,98 ; \mathrm{DE}=, 983)$.

\section{Prueba T para muestras relacionadas}

Se aplicó la prueba $\mathrm{T}$ para muestras relacionadas para observar si las diferencias de las medias de las cinco categorías evaluadas pre y pos cirugía eran estadísticamente significativas con un nivel de confiabilidad del $95 \%$.

\section{Categoría: Síntomas}

Se obtuvieron diferencias significativas entre los puntajes pre y pos cirugía para la categoría Síntomas $(\mathrm{T}=-22,97$; $p<0.001$ ).

\section{Categoría: Dolor}

Se obtuvieron diferencias significativas entre los puntajes pre y pos cirugía para la categoría Dolor $(\mathrm{T}=-28,86$; $p<0.001$ ).

\section{Categoría: Actividades cotidianas}

Se obtuvieron diferencias significativas entre los puntajes pre y pos cirugía para la categoría Actividades cotidianas $(\mathrm{T}=-35,96 ; p<0.001)$.

\section{Categoría: Función actividades deportivas y recreacionales}

Se obtuvieron diferencias significativas entre los puntajes pre y pos cirugía para la categoría Función actividades deportivas y recreacionales ( $\mathrm{T}=-26,06 ; p<0,001$ ).

\section{Categoría: Calidad de Vida}

Se obtuvieron diferencias significativas entre los puntajes pre y pos cirugía para la categoría Función actividades deportivas y recreacionales ( $\mathrm{T}=-25,83 ; p<0,001)$.

\section{Comentario}

Conseguir que los pacientes puedan acceder a procedimientos que permitan reducir variables intraoperatorias asociadas a un resultado negativo, ha sido el foco de la innovación tecnológica en esa área. Para poder concluir que existe una mejoría que extrapola las hipótesis técnicas, es necesario recurrir a los PROMs para evaluar los resultados clínicos.

La fortaleza de este estudio es en gran parte su simplicidad y la magnitud de la diferencia demostrada en los resultados pre y pos quirúrgicos. El hecho de que todos los pacientes incluidos en este trabajo fueron operados por el mismo cirujano, sumado a que se utilizó el mismo tipo de prótesis, ayuda a reducir la interferencia de variables externas al procedimiento. Lo que adquiere especial relevancia en las artroplastias de rodilla, dónde se ha observado que diferentes métodos y prótesis pueden cumplir un rol determinante en los resultados.

Pero este estudio descriptivo no está exento de debilidades, siendo algunas: el pequeño volumen de pacientes, el corto tiempo de estudio pos quirúrgico, y la ausencia de un grupo control.

Es importante resaltar que Reginato et al, observaron que en América Latina la articulación de la rodilla es la más frecuentemente afectada por la osteoartritis, Y se observó también que en Chile la presentación de la enfermedad se asocia radiológicamente con un grado de severidad menor que en el resto de los países latinoamericanos. ${ }^{38}$ Lo cual torna relevante la aplicación de terapias quirúrgicas más conservadoras que le ofrezcan al paciente un alivio sintomático y le permitan reestablecer sus actividades cotidianas causando el mínimo impacto posible.

Sería importante evaluar en un próximo estudio la relación entre la PROM de elección con variables como el índice de masa corporal, nivel socio económico, expectativas de mejoría y la etnicidad del paciente.

Se requieren más estudios al respecto para evaluar ventajas relacionadas a la longevidad de la prótesis, complicaciones a largo plazo y resultados en comparación a otros procedimientos actuales.

\section{Conclusión}

Este estudio evidenció que existe una diferencia estadísticamente relevante en el nivel de percepción de los síntomas de los pacientes sometidos a una artroplastia unicompartimental de rodilla mediante el sistema robótico NAVIO y el sistema protésico Stride (-Fig. 1). Todas las categorías evaluadas demostraron un aumento significativo en su puntaje; siendo un valor más alto y por lo tanto más satisfactorio (Tabla 1).

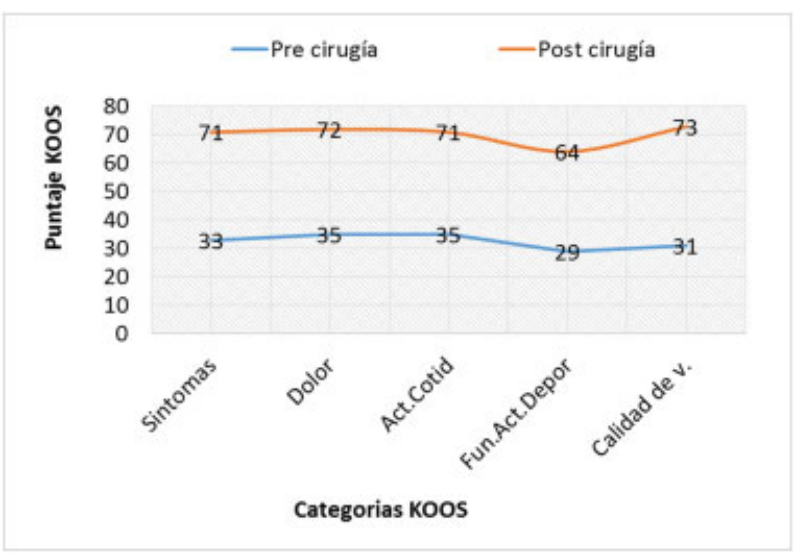

Fig. 1 Comparación en el nivel de percepción de los síntomas de los pacientes sometidos a una artroplastia unicompartimental de rodilla mediante el sistema robótico NAVIO y el sistema protésico Stride.

Abreviación: KOOS, Knee injury Osteoarthritis Outcome Score. 


\section{Referencias}

1 Mathers DSC, Pfleger B. Global burden of osteoarthritis in the year 2000http://cdrwww.who.int/entity/healthinfo/statistics/bod_osteoarthritis.pdf. Accessed May 20, 2016.

2 Branco JC, Rodrigues AM, Gouveia N, et al; EpiReumaPt study group. Prevalence of rheumatic and musculoskeletal diseases and their impact on health-related quality of life, physical function and mental health in Portugal: results from EpiReumaPt- a national health survey. RMD Open 2016;2(01):e000166

3 Murray CJL, Vos T, Lozano R, et al. Disability-adjusted life years (DALYs) for 291 diseases and injuries in 21 regions, 1990-2010: a systematic analysis for the Global Burden of Disease Study 2010. Lancet 2012;380(9859):2197-2223

4 Cisternas MG, Murphy LB, Yelin EH, Foreman AJ, Pasta DJ, Helmick CG. Trends in medical care expenditures of US adults with arthritis and other rheumatic conditions 1997 to 2005. J Rheumatol 2009;36(11):2531-2538

5 Kurtz S, Ong K, Lau E, Mowat F, Halpern M. Projections of primary and revision hip and knee arthroplasty in the United States from 2005 to 2030. J Bone Joint Surg Am 2007;89(04):780-785

6 Price AJ, Svard U. A second decade lifetable survival analysis of the Oxford unicompartmental knee arthroplasty. Clin Orthop Relat Res 2011;469(01):174-179

7 Laskin RS. Unicompartmental knee replacement: some unanswered questions. Clin Orthop Relat Res 2001;(392):267-271

8 Gooberman-Hill R, Sansom A, Sanders CM, et al. Unstated factors in orthopaedic decision-making: a qualitative study. BMC Musculoskelet Disord 2010;11(01):213

9 Hawker GA. Who, when, and why total joint replacement surgery? The patient's perspective. Curr Opin Rheumatol 2006;18(05):526-530

10 Cella D, Hahn EA, Jensen SE, et al. Patient-reported outcomes in performance measurement. RTI. September 2015https://www. rti.org/publication/patient-reported-outcomes-performancemeasurement. Accessed December 27, 2016.

11 Wylde V,Dieppe P, HewlettS, Learmonth ID. Total knee replacement: is it really an effective procedure for all? Knee 2007;14(06):417-423

12 Baker PN, Petheram T, Jameson SS, et al. Comparison of patientreported outcome measures following total and unicondylar knee replacement. J Bone Joint Surg Br 2012;94(07):919-927

13 Rana AJ. Building a Patient-Reported Outcome Metric Database: One Hospital's Experience. J Arthroplasty 2016;31(06):1151-1154

14 Lyman S, Hidaka C. Patient-Reported Outcome Measures-What Data Do We Really Need? J Arthroplasty 2016;31(06):1144-1147

15 Witjes S, Gouttebarge V, Kuijer PPFM, van Geenen RCI, Poolman RW, Kerkhoffs GMMJ. Return to Sports and Physical Activity After Total and Unicondylar Knee Arthroplasty: A Systematic Review and Meta-Analysis. Sports Med 2016;46(02):269-292

16 Hopper GP, Leach WJ. Participation in sporting activities following knee replacement: total versus unicompartmental. Knee Surg Sports Traumatol Arthrosc 2008;16(10):973-979

17 Newman JH, Ackroyd CE, Shah NA. Unicompartmental or total knee replacement? Five-year results of a prospective, randomised trial of 102 osteoarthritic knees with unicompartmental arthritis. J Bone Joint Surg Br 1998;80(05):862-865

18 Wiik AV, Manning V, Strachan RK, Amis AA, Cobb JP. Unicompartmental knee arthroplasty enables near normal gait at higher speeds, unlike total knee arthroplasty. J Arthroplasty 2013;28 (9, Suppl)176-178

19 Kim HJ, Yoon J-R, Choi GW, Yang J-H. Imageless Navigation Versus Conventional Open Wedge High Tibial Osteotomy: A Meta-Analysis of Comparative Studies. Knee Surg Relat Res 2016;28(01):16-26

20 Keene G, Simpson D, Kalairajah Y. Limb alignment in computerassisted minimally-invasive unicompartmental knee replacement. J Bone Joint Surg Br 2006;88(01):44-48
21 CobbJ,HenckelJ,GomesP, etal.Hands-on roboticunicompartmental knee replacement: a prospective, randomised controlled study of the acrobot system. J Bone Joint Surg Br 2006;88(02):188-197

22 Smith JR, Picard F, Lonner J, et al. The Accuracy of a RoboticallyControlled Freehand Sculpting Tool for Unicondylar Knee Arthroplasty. Bone Joint J 2014;96-B(SUPP 16):12-12

23 Lonner JH. Robotically Assisted Unicompartmental Knee Arthroplasty with a Handheld Image-Free Sculpting Tool. Oper Tech Orthop 2015;25(02):104-113

24 Hernigou P, Deschamps G. Alignment influences wear in the knee after medial unicompartmental arthroplasty. Clin Orthop Relat Res 2004;(423):161-165

25 Kim Y-H, Park J-W, Kim J-S, Park S-D. The relationship between the survival of total knee arthroplasty and postoperative coronal, sagittal and rotational alignment of knee prosthesis. Int Orthop 2014;38(02):379-385

26 Zhang GQ, Chen JY, Chai W, Liu M, Wang Y. Comparison between computer-assisted-navigation and conventional total knee arthroplasties in patients undergoing simultaneous bilateral procedures: a randomized clinical trial. J Bone Joint Surg Am 2011; 93(13):1190-1196

27 Methods and systems to control a shaping tool. April 2003https:// patents.google.com/patent/US6757582B2/en. Accessed December 26, 2016.

28 Lonner JH, Smith JR, Picard F, Hamlin B, Rowe PJ, Riches PE. High degree of accuracy of a novel image-free handheld robot for unicondylar knee arthroplasty in a cadaveric study. Clin Orthop Relat Res 2015;473(01):206-212

29 Gregori A, Picard F, Bellemans J, Smith J, Simone A. Handheld Precision Sculpting Tool for Unicondylar Knee Arthroplasty. A clinical Review. 4-6 June. http://bluebelttech.com/site/wp-content/pdfs/Clinical_Review_Handheld_Precision_Sculting_Tool. pdf. Accessed June 13, 2016.

30 Simons M, Riches P. The Learning Curve of Robotically-Assisted Unicondylar Knee Arthroplasty. Bone Joint J 2014;96-B(SUPP 11) 152-152

31 DEPARTMENT OF HEALTH \& HUMAN SERVICES F. 510(K) Summary NAVIO PFS. https://www.accessdata.fda.gov/cdrh_docs/ pdf12/K121936.pdf. Accessed November 14, 2016.

32 STRIDE Unicondylar Knee System | Smith \& Nephew - US Professional. http://www.smith-nephew.com/professional/products/all-products/stride/. Accessed June 14, 2016.

33 McEwen HMJ, Barnett PI, Bell CJ, et al. The influence of design, materials and kinematics on the in vitro wear of total knee replacements. J Biomech 2005;38(02):357-365

34 Stewart T, Jin ZM, Shaw D, Auger DD, Stone M, Fisher J. Experimental and theoretical study of the contact mechanics of five total knee joint replacements. Proc Inst Mech Eng H 1995;209(04):225-231

35 Bellamy N, Buchanan WW, Goldsmith CH, Campbell J, Stitt LW. Validation study of WOMAC: a health status instrument for measuring clinically important patient relevant outcomes to antirheumatic drug therapy in patients with osteoarthritis of the hip or knee. J Rheumatol 1988;15(12):1833-1840

36 Vaquero J, Longo UG, Forriol F, Martinelli N, Vethencourt R, Denaro V. Reliability, validity and responsiveness of the Spanish version of the Knee Injury and Osteoarthritis Outcome Score (KOOS) in patients with chondral lesion of the knee. Knee Surg Sports Traumatol Arthrosc 2014;22(01):104-108

37 Knee injury and Osteoarthritis Outcome Score, Ewa Roos. http:// koos.nu/. Accessed July 4, 2016.

38 Reginato AM, Riera H, Vera M, et al; Pan-American League of Associations for Rheumatology (PANLAR) Osteoarthritis Study Group. Osteoarthritis in Latin America: Study of Demographic and Clinical Characteristics in 3040 Patients. J Clin Rheumatol 2015;21(08):391-397 